\section{Paradoxical roles of mutational load as a determinant of anticancer immunity}

Immune-checkpoint inhibitors (ICIs)

have reshaped oncology practice,

but continued research is required to understand why only a minority of patients have durable responses and to better identify this subgroup. A high tumour mutational burden (TMB) has been identified as a key ...although a high TMB might predict successful treatment, excessive

ITH could actually have a negative effect...

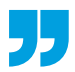

UVB-exposed cell line or mixtures of SCCs had greater tumorigenic activity. This effect was generally independent of TMB, but higher levels of genetic diversity, defined by low clonal to subclonal mutation ratios, were correlated with increased tumorigenicity. By contrast, the individual SCCs grew similarly to the original polyclonal cell line in immunodeficient mice, suggesting that antitumour immune responses limit tumorigenicity in the context of low levels of ITH. Analyses of tumour-infiltrating lymphocytes revealed greater numbers of $\mathrm{CD} 8^{+}$ $\mathrm{T}$ cells in the tumour core and evidence of increased cytolytic activity, and fewer regulatory $\mathrm{T}$ cells, in SCC-derived tumours than in polyclonal tumours. Notably, the clonal structure and heterogeneity of the polyclonal populations did not change over time, suggesting that their greater tumorigenic potential is not attributable to the presence of 'escaper' subclones.

"These findings were mirrored in retrospective data from patients with melanoma, which demonstrated that overall survival (OS) as well as responsiveness to ICIs is highly influenced by ITH,' Samuels states. Notably, TMB was not associated with patient survival in this analysis. Moreover, in a meta-analysis of four cohorts of patients treated with ICIs, a low Shannon diversity index, which incorporates both the number of clones and the diversity of the mutations across clones, was associated with better $O S(P=0.011)$, even after adjusting for TMB $(P=0.039)$.

These findings might explain why some tumours with a high TMB do not have an abundance of $\mathrm{T}$ cell infiltrates or respond to ICIs, whereas others with a low TMB do.
"The effect of ITH on the response of patients with melanoma to ICIs is probably no less important than that of TMB; although a high TMB might predict successful treatment, excessive ITH could actually have a negative effect and, therefore, the clonality of the tumour should be used as an additional biomarker to improve patient selection," explains Samuels.

"Our observations provide yet another important reminder of the complex challenges ahead of us; for example, therapeutic strategies to increase TMB have been proposed, but care is needed to ensure that ITH is not increased excessively in the process," adds Eytan Ruppin. Indeed, the results of this study support previous observations that clonal neoantigens are associated with favourable patient outcomes and sensitivity to ICIs, whereas subclonal neoantigens, including those generated by cytotoxic therapies, are associated with insensitivity to ICIs. "Seeing as our data supports the notion that clonal neoantigens are more likely to lead to better immune responses, we will use our model to design rational vaccination modalities tailored to the level of ITH and evaluate these with or without ICIs," says Samuels.

However, these findings raise many additional questions, as outlined by Ruppin: "For example, as ITH increases, does the tumour microenvironment change in a systematic manner? And does the timing of treatment with ICIs affect the development of ITH?"

To provide answers, the authors plan to use their innovative mouse model to gain a better mechanistic understanding of the effects of ITH on the immune response more broadly, beyond T cells, which might open new opportunities for improvements with ICIs.

David Killock

ORIGINAL ARTICLE Wolf, Y. et al. UVB-induced tumor heterogeneity diminishes immune response in melanoma. Cell 179, 219-235 (2019) 